\title{
NOTE ON DISCRIMINANTS OF BINARY QUADRATIC FORMS WITH A SINGLE CLASS IN EACH GENUS
}

\section{J. DEAN SWIFT}

When discriminants of binary quadratic forms have a single class in each genus, the number of representations function may be explicitly determined., ${ }^{1,2}$ The completeness of the lists ${ }^{1}$ of such discriminants is still an open question. Dickson, ${ }^{1}$ Townes, ${ }^{1}$ and Hall ${ }^{3}$ have given extensive lists of tests based on the fundamental criterion:

A. If and only if the discriminant possesses a primitive reduced form, $(a, b, c), c>a>b>0$, there is more than a single class in each genus.

These tests are based on the prime decomposition of numbers defined recursively from the discriminant, and are thus rather difficult to apply in screening all possible discriminants up to a large $N$ to check the completeness of the list. For this purpose, a simple linear congruence test capable of mechanical application would be far preferable. Accordingly, the following tests were developed:

B. A discriminant has more than a single class in each genus if:

a. $\Delta \equiv 7(\bmod 8)$ unless $\Delta=7$ or 15 . (Note: All odd $\Delta$ are congruent to 3 or 7 modulo 8 .)

b. $\Delta \equiv 12(\bmod 16)$ unless $\Delta=12,28$, or 60 .

$\Delta \equiv 0(\bmod 16)$ unless $\Delta=16,48,64,112,192,240,448,960$, or $\Delta \equiv 32(\bmod 64)$.

c. $\Delta \equiv 0\left(\bmod \mathrm{p}^{2}\right), p$ an odd prime, unless $\Delta=27,36,72,75,99$, $100,147,180,288,315$.

d. $\Delta$ is a negative quadratic residue of an odd prime, $p$, such that $p^{2}<\left(\Delta+\beta^{2}\right) / 4$, where $\beta$ is the largest number less than $p$ of the same parity as $\Delta$.

Test (a) is proved by Dickson ${ }^{1}$ and tests (b) and (c) by Hall. ${ }^{3}$ For test (d) assume $\Delta \equiv-b^{2}(\bmod p)$. Then $\Delta+b^{2}=4 m p, m>p>b$. Thus the representation, $(p, b, m)$, exists, satisfying A. Possible imprimitive forms are taken care of by test (c).

Test (d) is essentially similar to that used by Lehmer ${ }^{4}$ in checking the completeness of the "class number one" tables to $5,000,000,000$.

Received by the editors July 29, 1947.

${ }^{1}$ L. E. Dickson, Introduction to the theory of numbers, University of Chicago, 1929, chap. 5, pp. 63-90. The notation and terms used herein are as defined in this work.

2 N. A. Hall, Amer. J. Math. vol. 62 (1940) pp. 589-598.

${ }^{8}$ N. A. Hall, Math. Zeit. vol. 44 (1938) pp. 85-90. Further references to this and associated problems will be found in these papers of Hall.

- D. H. Lehmer, Bull. Amer. Math. Soc. Abstract 39-5-188. 
The $T_{k}$ and $S_{k}$ tests given by Dickson, Townes, and Hall are derivable from these tests by consideration of the requirements imposed by test (d) on the $T_{k}$ and $S_{k}$.

Using a small linear congruence machine developed by $\mathrm{D}$. $\mathrm{H}$. Lehmer, and with the kind assistance of Prof. and Mrs. Lehmer, the author checked the possible discriminants to $10^{7}$, verifying the following theorem.

THEOREM: There are no discriminants with a single class in each genus, $3315<\Delta<10,000,000$.

The largest prime necessary in this test was 79.

California Institute of Technology

\section{ON FINITE EXTENDING GROUPS}

\section{ALBERT NEWHOUSE ${ }^{1}$}

In his paper Non-associative algebras, ${ }^{2}$ A. A. Albert defined extending groups $\mathbb{B}$ for algebras $\mathfrak{A}$ with a unity element. ${ }^{3}$ Such groups are merely finite multiplicative groups of nonsingular linear transformations on a linear space $\mathfrak{A}$ of order $n>1$ over a field $\mathfrak{F}$ defined so that all the transformations leave the unity element $e$ of $\mathfrak{A}$ unaltered. With respect to the basis $\left(e, u_{2}, u_{3}, \cdots, u_{n}\right)$ of $\mathfrak{A}$ over $\mathfrak{F}$ these groups are then isomorphic to finite groups $(B)$ of $n$-rowed square matrices of the form

$$
G=\left(\begin{array}{ll}
1 & 0 \\
B & M
\end{array}\right)
$$

where $M$ is an $(n-1)$-rowed nonsingular square matrix and $B$ a 1 by $n-1$ matrix.

In his paper Albert ${ }^{4}$ has raised the question of the existence of such groups (\&) "such that no basis of $\mathfrak{A}$ exists for which \& may be regarded as a permutation group."

Presented to the Society, April 29, 1944; received by the editors March 14, 1944, and, in revised form, April 18, 1947, and July 29, 1947.

1 The author is indebted to the referee for his helpful comments.

${ }^{2}$ Ann. of Math. vol. 43 (1942) pp. 685-723.

8 Ibid. p. 712.

4 Ibid. Footnote, p. 722. 